\title{
Functional analysis of the Epichloë festucae-perennial ryegrass symbiosis
}

\author{
B. SCOTT ${ }^{1,2}$, D. TAKEMOTO ${ }^{1,2}$, A. TANAKA ${ }^{1}$, C.A. YOUNG ${ }^{1,3}$, M.K. BRYANT ${ }^{1}$ and K.J. MAY ${ }^{1,2}$ \\ ${ }^{1}$ Centre for Functional Genomics, Institute of Molecular BioSciences, Massey University, Private Bag 11222 , \\ Palmerston North, New Zealand \\ ${ }^{2}$ National Centre for BioProtection, Massey University, Private Bag 11 222, Palmerston North, New Zealand \\ ${ }^{3}$ Forage Improvement Division, The Samuel Roberts Noble Foundation, Inc. 2510 Sam Noble Pky, Ardmore, OK 73401, USA.
}

d.b.scott@massey.ac.nz

\begin{abstract}
Although much is known about the beneficial and detrimental effects of the interaction between Neotyphodium lolii and Lolium perenne in the field comparatively little is known about the molecular and cellular events that underlie these effects and how mutualism is maintained. Because $N$. lolii is quite intractable to genetic analysis we have developed the synthetic association between Epichloë festucae and perennial ryegrass as our model experimental system to study the epichloë endophytegrass symbiotic interaction. Using this system we have recently shown that reactive oxygen species (ROS) produced by a specific endophyte NADPH oxidase (NoxA) have a critical role in regulating hyphal growth and development in perennial ryegrass. We have also shown that two additional components, NoxR and RacA, are required to regulate ROS production to maintain a mutualistic interaction. Using a combined molecular and genetic approach we have recently cloned and characterised genes for peramine and lolitrem biosynthesis. An overview of these advances and the opportunities now available to better
\end{abstract}

understand and exploit this important fungal-grass interaction is presented.

Keywords: Epichloë festucae, Neotyphodium lolii, Lolium perenne, peramine, lolitrems, reactive oxygen species

\section{Introduction}

Neotyphodium lolii is an obligate seed-borne biotrophic fungus that colonises the intercellular spaces of perennial ryegrass aerial tissues, including the vegetative and reproductive tillers, as well as the seed (Christensen et al. 2002; Philipson \& Christey 1986). Hyphae ramify within the meristematic zone of the grass from where they colonise axillary buds and leaf primordia. The hyphae in the leaves are infrequently branched, grow parallel to the axis of the leaf, and have a growth pattern that is synchronised with that of the host throughout the life cycle of the grass (Christensen et al. 2002; Tan et al. 2001). Strict control of hyphal growth is critical for maintenance of the mutualistic interaction between endophyte and grass host (Tanaka et al. 2006).

N. lolii is a haploid asexual derivative of Epichloë festucae, a

Table 1 Frequency of homologous gene replacement in E. festucae

\begin{tabular}{|c|c|c|c|c|c|}
\hline GENE & $\begin{array}{c}5^{\prime} \text { flank } \\
(\mathrm{kb})\end{array}$ & $\begin{array}{c}3^{\prime} \text { flank } \\
\text { (kb) }\end{array}$ & $\% \mathrm{KO}$ & Number & Reference \\
\hline ItmM & 2.7 & 2.7 & 3.1 & 5 of 159 & Young et al. (2005) \\
\hline nox $B^{1}$ & 1.8 & 1.2 & 5.0 & 1 of 20 & Tanaka et al. (2006) \\
\hline $\operatorname{nox} B^{2}$ & 1.8 & 1.2 & 2.5 & 1 of 40 & Tanaka et al. (2006) \\
\hline $\operatorname{nox} A$ & 2.5 & 0.8 & 3.7 & 2 of 54 & Tanaka et al. (2006) \\
\hline perA & 2.6 & 2.5 & 0.8 & 1 of 120 & Tanaka et al. (2005) \\
\hline $\operatorname{sak} A$ & 3.2 & 1.5 & 8.3 & 1 of 12 & Eaton unpublished results \\
\hline$g c n A$ & 2.8 & 2.8 & 10.0 & 2 of 20 & Bryant et al. (2007) \\
\hline ItmJ & 1.4 & 1.0 & 8.3 & 3 of 36 & Takemoto unpublished results \\
\hline ItmF & 1.3 & 3.5 & 8.3 & 1 of 12 & Takemoto unpublished results \\
\hline ItmE & 1.2 & 1.5 & 1.0 & 1 of 96 & Takemoto unpublished results \\
\hline ItmK & 1.6 & 1.2 & 16.7 & 2 of 12 & Takemoto unpublished results \\
\hline $\operatorname{Itm} Q$ & 1.8 & 1.7 & 1.3 & 1 of 80 & Takemoto unpublished results \\
\hline $\operatorname{noxR}$ & 1.5 & 2.5 & 25.0 & 6 of 24 & Takemoto et al. (2005) \\
\hline $\operatorname{rac} A$ & 1.8 & 0.9 & 1.0 & 1 of 100 & Tanaka unpublished results \\
\hline EF108 & 1.1 & 1.7 & 6.6 & 2 of 30 & Tanaka et al. (2005) \\
\hline $\mathrm{Itm}^{3}$ & 2.7 & 2.7 & 4.0 & 4 of 100 & May unpublished results \\
\hline
\end{tabular}

${ }^{1}$ In wt background

${ }^{2}$ In noxA background

${ }^{2}$ Knock-in 
Figure 1 Symbiotic phenotype of E. festucae noxA. Phenotypes of perennial ryegrass infected with $E$. festucae wild-type Fl1 and noxA symbiotic mutant.

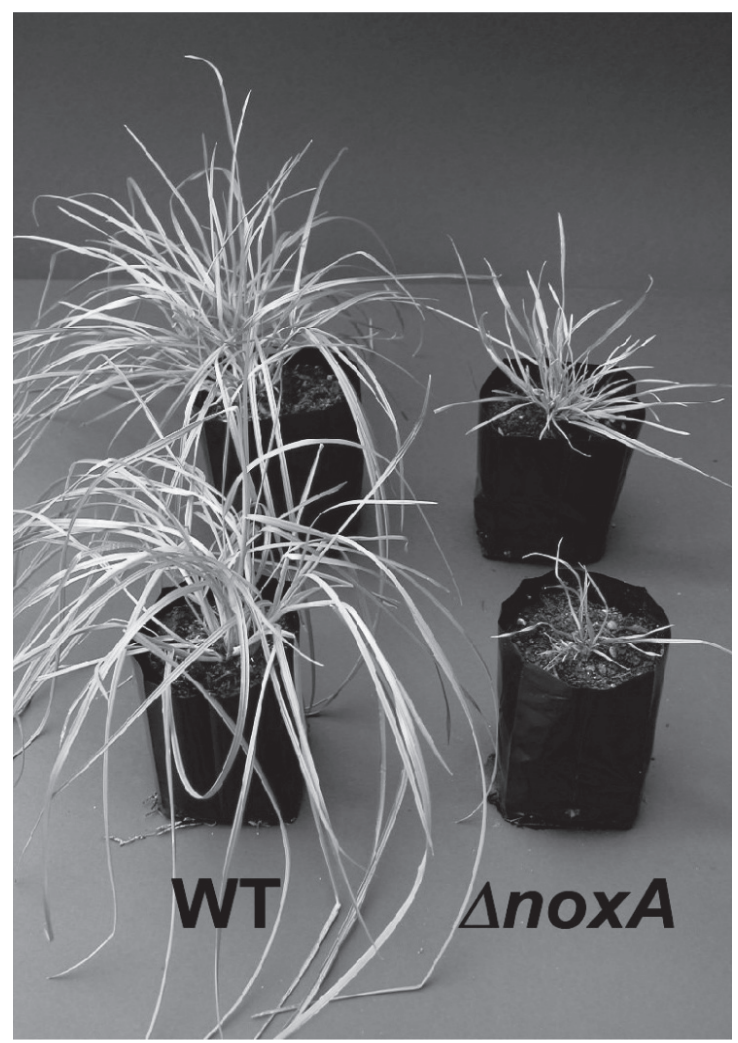

natural symbiont of Festuca spp. that is also capable of forming compatible associations with perennial ryegrass (Christensen et al. 1997; Leuchtmann et al. 1994). Numerous studies have established that $N$. lolii, E. festucae and related epichloë endophytes are mutualists that provide a wide range of important benefits to their host plants, particularly under conditions of biotic or abiotic stress (Clay 1990; Easton 1999; Schardl \& Clay 1997). The most well documented benefit of $N$. lolii to the perennial ryegrass host is protection from insect herbivory (Ball et al. 1997; Prestidge \& Gallagher 1988; Rowan \& Gaynor 1986), a biological effect ascribed to endophyte synthesis of various alkaloids in planta.

Three main classes of biologically active metabolites have been identified in $N$. lolii infected perennial ryegrass: peramine, the only known pyrrolopyrazine; ergot alkaloids, principally ergovaline; and indole-diterpenes, principally lolitrem B (Lane et al. 2000; Rowan 1993). Peramine has been shown to be a potent feeding deterrent of adult Argentine stem weevil (ASW; Listronotus bonariensis) (Rowan et al. 1990; Rowan \& Gaynor 1986), an economically important exotic pest of perennial ryegrass in New Zealand. Lolitrem B has biological activity against ASW larvae (Prestidge \& Gallagher 1988) but is better known as the causative agent of the neuromuscular disorder known as ryegrass staggers, associated with sheep grazing ryegrass dominant pastures after long periods of water stress or following fresh re-growth after periods of water stress (Fletcher \& Easton 1997; Keogh 1973). The importance of ergot alkaloids toward ecological fitness
Figure 2 Models for regulation of ROS production by Nox complex in culture and in planta. (A) Model for regulation of NoxA-catalysed ROS production in culture by recruitment of RacA and other unidentified proteins to the endophyte plasma membrane in response to nutritional or environmental signals. (B) Model for regulation of NoxAcatalysed ROS production in planta by recruitment of RacA and NoxR to the endophyte plasma membrane in response to a signal from the host grass.

\section{In culture}

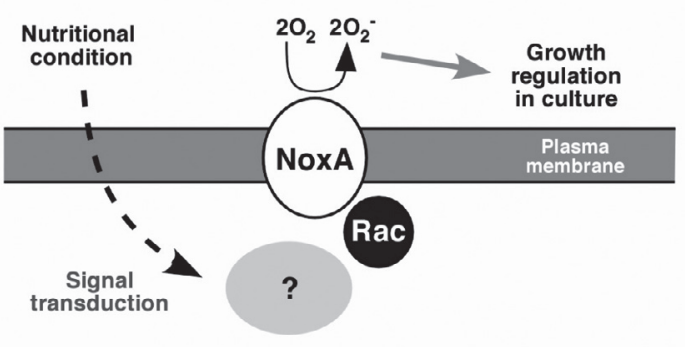

\section{In host plant}

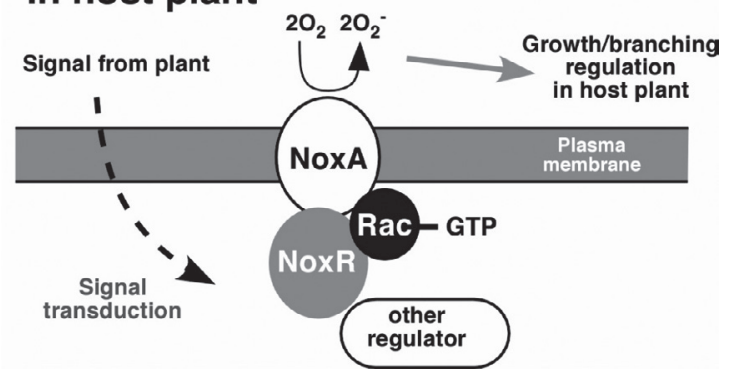

of the host grass has not been well defined. The presence of ergopeptines in perennial ryegrass potentially exacerbates ryegrass staggers, as they induce heat stress in grazing livestock (Fletcher \& Easton 1997). Alkaloid levels in endophyte-infected perennial ryegrass are affected by season (Ball et al. 1995; di Menna \& Waller 1986), environment (Barker et al. 1993; Lane et al. 1997; Rasmussen et al. 2006) and plant genotype (Easton et al. 2002; Latch 1994; Spiering et al. 2005).

Genes for the biosynthesis of ergot alkaloids (Panaccione et al. 2001; Wang et al. 2004), indole-diterpenes (Young et al. 2005; Young et al. 2006) and peramine (Tanaka et al. 2005) have now been cloned. Expression analysis has shown that the genes for all three pathways are preferentially and highly expressed in planta (Tanaka et al. 2005; Young et al. 2006), suggesting that plantspecific signalling is required for expression of these pathways. The molecular cloning of these genes now allows the biosynthetic pathways to be elucidated, the mechanisms for plant-regulated expression to be explored, and the functional distribution of these genes within the epichloë endophytes to be determined.

The recent demonstration that endophyte production of reactive oxygen species (ROS) is critical in maintaining the mutualistic interaction between $E$. festucae and perennial ryegrass provides important insights into potential signalling mechanisms that operate between endophyte and host (Takemoto et al. 2006; Tanaka et al. 2006). The isolation and characterisation of the fungal components of the NADPH oxidase complex and associated regulators and effectors now allows key signalling pathways to be elucidated. 
Figure 3 Design of perA replacement construct pPN66.

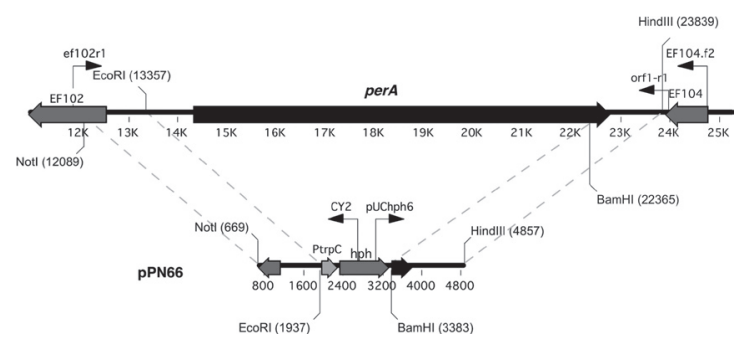

Figure 5 Physical map of the N. Iolii (Lp19) and E. festucae (Fl1) LTM locus. The map of the Lp19 LTM locus was generated from sequence accession numbers AY742903 and DQ443465. The map of the Fl1 LTM locus was generated from sequence accession number AY742905 and from Southern analysis.

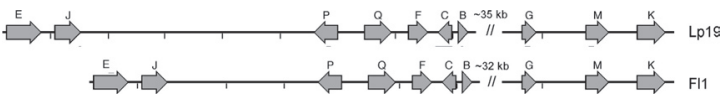

This review provides an overview of recent advances in our understanding of (i) endophyte signalling mechanisms that maintain the mutualistic symbiotic interaction between $E$. festucae and perennial ryegrass and (ii) the endophyte genes and gene products required for biosynthesis of peramine and lolitrems.

\section{Methods}

\section{Fungal strains and growth conditions}

Cultures of Epichloë festucae wild-type strain F11 and mutant strains were grown on $2.4 \%$ potato dextrose (PD) agar at $22^{\circ} \mathrm{C}$.

Plant growth and endophyte inoculation conditions

Inaoculation of endophyte-free seedlings of perennial ryegrass (Lolium perenne) was performed by the method of Latch \& Christensen (1985). Plants were grown and tested for the presence of endophyte as described previously (Tanaka et al. 2005).

\section{Microscopy}

Fungal hyphae were examined using the Zeiss Axiophot microscope with DIC optics. Images were captured using IM50 (version 4.0) image capture software. The presence of hyphae in endophyte-infected leaves was examined by staining of epidermal peels from outer leaf sheaths with an aniline blue stain. An Olympus BX51 light microscope was used to examine the hyphal structure, with images captured by an Optronics digital camera using the MagnaFire 2.1C image capture software.

\section{GUS analysis}

Plants inoculated with $E$. festucae isolates transformed with the $\mathrm{PltmM}$-gusA and PgpdA-gusA reporter constructs were stained for GUS activity as described previously (Bryant et al. 2007).

\section{Preparation of constructs}

Plasmid pPN63 was prepared by sequentially ligating into pPN1688 (Young et al. 2005) a 1.8-kb KpnI/SacI (3') and a 2.0$\mathrm{kb}$ PstI/SalI (5') fragment from gene EF101. The two fragments were prepared by cloning $1.8 \mathrm{~kb} K p n \mathrm{I} / \mathrm{NotI}$ and $2.0-\mathrm{kb} E c o \mathrm{RV}$ fragments from pPN60 into pBlueScript and redigesting to capture appropriate restriction enzyme sites that were part of the multiple cloning site.
Figure 4 A proposed scheme for peramine biosynthesis. Adaptated from Tanaka et al. (2005).

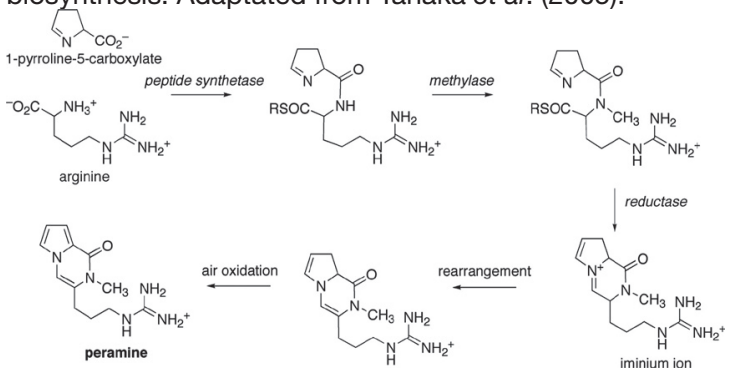

Plasmid pPN66 was prepared by sequentially ligating into pPN1688 (Young et al. 2005) 1.94-kb NotI/EcoRI (5') and a 1.47-kb BamHI/HindIII (3') fragments from the 5' end of EF102 and the 3 ' end of perA, respectively.

\section{Results and Discussion}

Epichlö festucae as a model experimental system for genetic analysis of endophytes

Although $N$. lolii is the natural epichloë endophytic species found in perennial ryegrass it is quite intractable to genetic analysis principally because of its extremely slow growth rate in axenic culture. In contrast, E. festucae is relatively fast growing, forming large $(1.5-\mathrm{cm})$ colonies on potato dextrose agar plates within a period of 2 weeks, compared to six-ten weeks for $N$. lolii. Hyphae of $E$. festucae readily form protoplasts when treated with Glucanex and as long as the protoplast viable count is at least $10^{6} /$ $\mathrm{ml}$, good transformation rates are usually obtained (Tanaka et al. 2005; Young et al. 2005). Using E. festucae strain Fl1, an isolate from Festuca longifolia seedline SR3000 (Siegel et al. 1990), targeted replacements can be generated in the genome of this strain by homologous recombination at frequencies of between 1 and $25 \%$ depending on the locus targeted and the size of the flanking sequences used to prepare the construct (Table 1). We generally use flanking sequences in the range of 1.5 to $2.5-\mathrm{kb}$ to maximise the yield of recombinants. E. festucae is naturally haploid with a genome size of approximately $29 \mathrm{Mb}$ (Kuldau et al. 1999), and has a heterothallic mating system (Leuchtmann et al. 1994). Synthetic associations between E. festucae strain Fl1 and perennial ryegrass can be readily established, thereby allowing genetic manipulation of this strain in culture and subsequent reintroduction into the host to study the symbiotic phenotype (Bryant et al. 2007; Takemoto et al. 2006; Tanaka et al. 2006; Tanaka et al. 2005; Young et al. 2005). In contrast to N. lolii, E. festucae is relatively easy to inoculate into perennial ryegrass seedlings with frequencies of infection in the range of 80 to $90 \%$ being routinely obtained with the wild-type strain. Although the growth of E. festucae Fl1 in these synthetic associations is slightly more vigorous than $N$. lolii, the hyphae still grow parallel to the axis of the leaf, are infrequently branched, and the pattern of growth is synchronised with that of the host throughout the life cycle of the grass (Takemoto et al. 2006; Tanaka et al. 2006). An important difference between N. lolii and E. festucae is the ability of the latter to form epiphyllous hyphae on the surface of perennial ryegrass leaves, but the growth of these hyphae are still tightly regulated by the host (Tanaka et al. 2006). Because of the above attributes, we have adopted E. festucae F11 and $L$. perenne as our model experimental system to study the epichloë endophyte-grass symbiotic interaction. 
Figure 6 Physical maps of the N. Iolii (Lp19) and E. festucae (Fl1) Itm cluster 1. The functional genes, abbreviated to a single letter, are represented by arrows indicating the direction they are transcribed. The microsatellite is represented by an asterisk. Tahi and Rua are relic retrotransposon sequences.

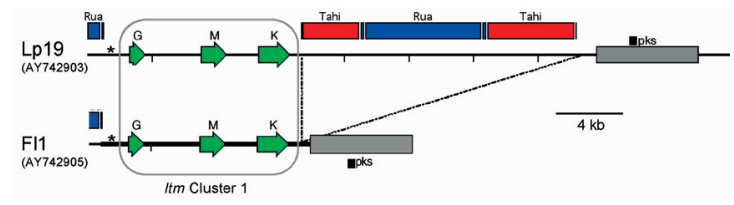

Figure 8 Analysis of GUS activity patterns of E. festucae hyphae infecting perennial ryegrass tillers using bright field microscopy. GUS expression patterns observed in infected, A, leaf sheath of vegetative tillers; B, preanthesis spikelets and individual florets; C, post-anthesis spikelets and individual florets; and, D, germinating seeds and seedlings. In panels $\mathrm{A}-\mathrm{C}$, photographs represent either PItmM-gusA transformants (column I) or Pgpd-gus $A$ transformants, the positive control (column II). Panel D are representative photographs of P/tmM-gusA transformants infecting perennial ryegrass seeds and seedlings.

\section{PltmM-gusA transformants II. Pgpd-gusA transformants}
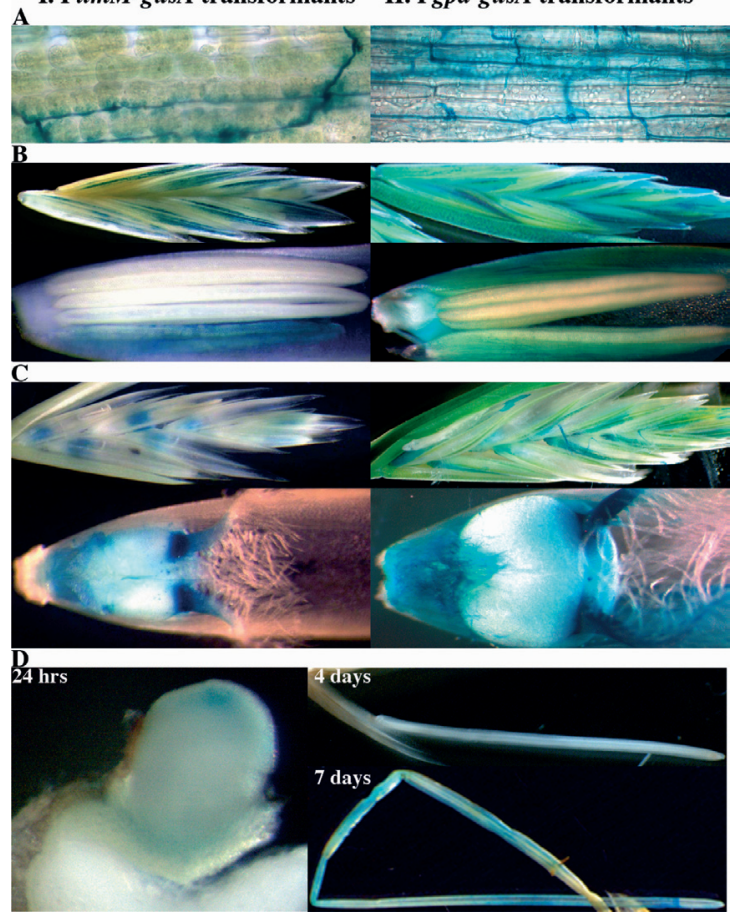

\section{Isolation of Epichloë festucae symbiotic mutants}

Epichloë festucae insertional mutagenesis

To identify E. festucae genes that are required for the establishment and maintenance of a mutualistic interaction with perennial ryegrass we have initiated a forward genetics approach (mutant phenotype to gene) to isolate mutants that disrupt this highly regulated symbiosis. Two insertional mutagenesis methods are being used, restriction enzyme-mediated plasmid integration
Figure 7 Structures of paspaline and lolitrem B.

A<smiles>CC(C)(O)[C@@H]1CC[C@@]2(C)C(CC[C@H]3Cc4c([nH]c5ccccc45)[C@]32C)C1(C)C</smiles>

B

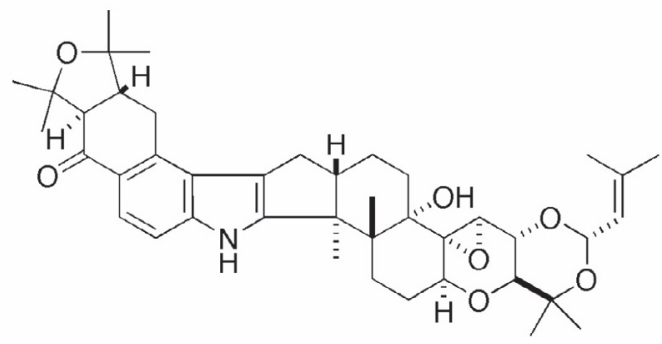

(REMI) (Schiestl \& Petes 1991) and Agrobacterium tumefaciens mediated T-DNA integration (Bundock et al. 1995; de Groot et al. 1998). The first method involves polyethylene glycol mediated uptake of linearised pAN7-1 plus restriction enzyme, into protoplasts of E. festucae (Tanaka et al. 2006). The presence of the restriction enzyme generates double stranded DNA breaks that promote recombination and repair of the plasmid into the fungal genome. The potential sites for integration are limited only by the number of sites in the genome for the restriction enzyme used. The major disadvantages of this method are the generation of multiple-copy and multi-site integrations and the generation of untagged deletions in the fungal genome (Michielse et al. 2005; Young et al. 2001). The second method involves acetosyringone activated conjugal transfer of T-DNA, engineered to contain a hygromycin resistant $(h p h)$ cassette, from A. tumefaciens to fungal cells (Bundock et al. 1995; de Groot et al. 1998). The development of this method using mycelial fragments of $E$. festucae is described in an accompanying paper (Tanaka et al. 2007). While this method preferentially results in single-site, single-copy integrations, there is a bias toward insertions into promoters of the fungal genes (Walton et al. 2005).

The use therefore of the combined approaches of REMI and T-DNA mediated insertional mutagenesis should maximise our ability to isolate mutations throughout the genome of E. festucae. The long-term goal is to generate sufficient number of mutants to provide widespread coverage of the E. festucae genome.

\section{Isolation and functional analysis of noxA}

Using REMI plasmid pAN7-1 mutagenesis we recently isolated a mutant of $E$. festucae that was symbiotically defective (Tanaka et al. 2006). This mutant was shown to have a single-copy insertion of pAN7-1 in the coding region of an NADPH oxidase gene, designated nox $A$, that is a homologue of the mammalian gp91phox, an NADPH oxidase that catalyses the conversion of molecular oxygen to superoxide. Plants infected with the noxA mutant are severely stunted and undergo precocious senescence (Fig. 1). Inactivation of this endophyte gene resulted in unregulated growth of the fungal hyphae in meristematic and mature leaf tissue resulting in a dramatic increase in fungal 
biomass in all tissues (Tanaka et al. 2006). In contrast to wildtype cells, hyphal cells of the noxA mutant showed an increase in vacuolation. Introduction of a wild-type copy of the gene into the nox $A$ mutant restored the ability of this strain to form a wild-type symbiotic interaction, confirming that the plasmid insertion in nox $A$ was responsible for the mutant phenotype. Deletion of a second NADPH oxidase gene, noxB, had no effect on the E. festucae-perennial ryegrass symbiotic interaction phenotype. Using transmission electron microscopy to locate electron-dense cerium perhydroxide deposits, we were able to show that $\mathrm{H}_{2} \mathrm{O}_{2}$ production was significantly reduced in the endophyte extracellular matrix and associated plant cell walls of meristematic tissue infected with the nox $A$ mutant, compared to wild-type. These results demonstrate that endophyte production of ROS, catalysed by a specific NADPH oxidase isoform, is critical for maintaining the mutualistic interaction between $E$. festucae and perennial ryegrass.

\section{Isolation and functional analysis of nox $R$ and racA}

Generation of ROS by the NADPH oxidase found in mammalian neutrophils requires formation of a multi-enzyme complex composed of the catalytic subunit gp91phox and the regulatory subunits p22phox, p40phox, p47phox, p67phox and the small GTPase Rac (Diebold \& Bokoch 2001; Lambeth 2004). Bioinformatic analysis of fungal genome sequences identified a gene encoding a protein with an $\mathrm{N}$-terminal domain very similar to $\mathrm{p} 67$ phox, which we have designated noxR, with motifs for both RAC and gp91phox binding (Takemoto et al. 2006). This gene was cloned from E. festucae and a deletion mutant generated by targeted gene replacement (Table 1). The plant interaction phenotype of symbiota containing the noxR mutant was very similar to that observed for the nox $A$ mutant i.e. plants were stunted and underwent rapid senescence (Takemoto et al. 2006). Light and confocal microscopic examination of leaf tissues revealed that associations containing the noxR mutant had an increase in hyphal biomass. In order to understand the role of NoxA and NoxR in regulating hyphal branching in planta, two experiments were carried out in culture that recapitulated the hyperbranching phenotype observed for the nox $R$ mutant in the plant. Overexpression of noxR, under the control of a TEF promoter, or depletion of ROS by growing wild-type cultures in the presence of diphenylene iodonium, disrupted normal apical tip growth and induced hyphal hyperbranching. These results indicate that NoxR has a crucial regulatory role in controlling hyphal tip growth and branching in the grass host. However, NoxR alone is insufficient to activate NoxA in planta. By analogy with the mammalian phagocytic system, which requires both p67phox and Rac2 for gp91phox activation, E. festucae also requires a small GTP binding protein, RacA, for ROS production. Yeast two-hybrid and pull-down assays showed NoxR interacts with RacA. A single amino acid substitution in the predicted RacA binding site of NoxR (R101E) abolished the ability of NoxR to complement a noxR mutation in planta, indicating that both NoxR and RacA are required to activate NoxA in the host plant.

These results suggest that localised ROS production, catalysed by NoxA, is critical for controlling the highly regulated growth pattern observed for E. festucae in the meristematic and vegetative tissue of the perennial ryegrass host. The genetic evidence suggests that NoxR and RacA control the spatial and temporal activation of NoxA in planta, and by extension hyphal tip growth and branching.

\section{Model for ROS regulation of hyphal growth in planta}

Based on our functional analysis of NoxA and NoxR we have developed a model to explain how these proteins control polarised growth of E. festucae in culture and in planta (Fig. 2). Maintenance of polarised growth of E. festucae in axenic culture is proposed to involve bursts of ROS catalysed by NoxA following recruitment of RacA and other potential regulators of Nox in response to environmental/nutritional cues. Growth of $E$. festucae in the symbiosis is proposed to involve recruitment of NoxR and RacA to the plasma membrane and activation of NoxA in response to plant signals.

Although we have established a role for ROS in regulating hyphal growth in the E. festucae-perennial ryegrass association many questions remain unanswered. Are there unique components of the Nox complex yet to be identified that are required for Nox activation in response to plant signalling? What is the mechanism for activation and recruitment of NoxR and RacA to the plasma membrane? What is the molecular form of the ROS signal and how is that signal perceived and transduced within the cell? The goal of current research is to provide answers to some of these questions.

\section{Isolation and functional analysis of genes for the synthesis of bioprotective metabolites}

Molecular cloning and functional analysis of a peramine biosynthetic gene

The structure of peramine, a pyrrolopyrazine, suggests that it is the product of a reaction catalysed by a two-module nonribosomal peptide synthetase (NRPS) that utilises proline and arginine as substrates. The conservation of polypeptide sequence in the adenylation (A) domain of known NRPSs has made it possible to use PCR strategies to clone various NRPSs from clavicipitalean fungi, including the lysergyl peptide synthetase (lpsA) required for ergovaline biosynthesis (Panaccione 1996; Panaccione et al. 2001). Using a similar strategy, candidate sequences for a peramine synthetase were amplified by RT-PCR using total RNA from $N$. lolii-infected perennial ryegrass (Tanaka et al. 2005). Four unique NRPS products were identified, two of which were weakly expressed in axenic culture but strongly expressed in planta. One of these sequences, clone ps9, crosshybridised to genomic DNA from epichloë endophytes known to synthesise peramine in planta. This probe was used to isolate a genomic clone from a pMOcosX cosmid library to E. festucae, strain F11. Sequence analysis of pPN60, a representative cosmid from the set isolated, identified a gene, which we have designated perA (EF103), predicted to encode a two-module NRPS. Nine additional genes (EF100-EF102, EF104-EF109), which show striking conservation of microsynteny with Fusarium graminearum and other filamentous fungal genome sequences, were identified on the perA-containing cosmid. The absence of per $A$ from the other fungal genomes analysed suggests that perA has been acquired in E. festucae by an insertional event in the intergenic region between EF102 and EF104. The presence of 12 bp direct repeats flanking perA supports this hypothesis. A replacement construct (pPN61) containing 1.68-kb of the T1 and half of the $\mathrm{C} 1$ domains of perA replaced with a hygromycin resistance cassette was prepared and recombined into the genome of F11. PCR analysis of the isolated transformants, identified one mutant (strain PN2323) out of 120 that contained the desired gene replacement (Table 1). Perennial ryegrass symbiota containing the perA mutant lacked detectable levels of peramine. In contrast, the levels of lolitrem B and ergovaline were similar to those found in wild-type symbiota. A wild-type copy of perA 
complemented the deletion mutant. Taken together these results confirm that perA is a NRPS for peramine biosynthesis.

In the process of doing these experiments it was observed that the perennial ryegrass infection rate for the perA mutant was considerably less than that observed for wild-type inoculations, and plants that were infected with the perA mutant frequently showed a stunted phenotype. However, some plants infected with the perA mutant had a wild-type symbiotic interaction phenotype. Re-isolates (e.g. PN2425) from these plants retained the wild-type symbiotic interaction phenotype when reinoculated into perennial ryegrass seedlings. However, these isolates were shown in axenic culture growth tests to be hygromycin sensitive. Molecular analysis revealed that PN2425 was missing the hygromycin resistance cassette and approximately 4-kb of perA sequence, indicating that the original mutant, strain PN2323, had undergone a secondary recombination event that resulted in deletion of the selectable marker and additional flanking per $A$ sequences. This analysis suggests that either the insertional (multiple copies of the $h p h$ cassette) and/or deletional (around the T1 domain of perA) event associated with the PN2323 gene replacement lead to a break-down in the mutualistic interaction between E. festucae and its perennial ryegrass host. To test this hypothesis a new gene replacement construct (pPN66), that removes almost the entire per $A$ was prepared and recombined into the genome of F11 (Fig. 3). PCR analysis of these transformants identified one, strain PN2468, out of 30 screened that contained a replacement. Infection rates for this mutant on perennial ryegrass seedlings were similar to that of Fl1. Symbiota containing the new perA mutant had a wild-type symbiotic interaction phenotype and were unable to synthesise peramine.

To determine whether additional genes at the perA locus were required for peramine biosynthesis, replacement constructs were prepared to EF101 (pPN63) and EF108 (pPN64), genes encoding putative reductase and dioxygenase functions, respectively. Symbiota containing an E. festucae EF108 replacement mutant had levels of peramine that were comparable to wild-type and ectopic controls, indicating that EF108 is not required for peramine biosynthesis. Attempts to isolate a replacement of EF101 were unsuccessful, despite screening $205 \mathrm{Hyg}^{\mathrm{R}}$ transformants. These results suggest that EF101 is essential for growth of $E$. festucae. Bioinformatic analysis predicts that EF101 encodes a 3-ketosteroid reductase for sterol biosynthesis, a function that would be indispensable for E. festucae growth.

Based on these results a scheme for the biosynthesis of peramine has been proposed that involves PerA alone (Tanaka et al. 2005). The primary substrates for peramine biosynthesis are proposed to be 1-pyrroline-5-carboxylate, a proline intermediate, and arginine (Fig. 4). We do not favour the use of proline as a substrate as additional enzyme functions would be needed for formation of the pyrrole ring. The condensation domain of PerA is proposed to catalyse formation of the peptide bond between 1-pyrroline-5-carboxylate and arginine. The methylation domain of PerA is proposed to catalyse the $N$-methylation of the amino group of arginine. The reductase domain is proposed to reduce the thioester and cyclise the dipeptide to form an iminium ion that is concomitantly released. Deprotonation of this intermediate and oxidation of the pyrroline ring would give rise to peramine.

The cloning of perA from E. festucae now makes it possible to investigate how this gene is regulated in the plant. The availability of an F11 perA isogenic mutant will allow us to test directly the role of peramine as a feeding deterrent to a range of insects, as has already been demonstrated for Argentine stem weevil (Tanaka et al. 2005), and carry out contained greenhouse or field trials to test the ecological benefits of this gene to the symbiotium. Using primers designed to different regions of the perA gene, a PCR test is now available to assess the peramine biosynthetic capability of epichloë endophytes (Scott et al. 2007).

Molecular cloning and functional analysis of genes for indolediterpene biosynthesis

A complex lolitrem biosynthetic locus (LTM) of at least 10 genes, organised in three mini-clusters, has been cloned and characterised from $N$. lolii strain Lp19 and E. festucae strain F11 (Young et al. 2005; Young et al. 2006) (Fig. 5). The first cluster contains three genes, $\operatorname{ltm} G$, ltm $M$ and $\operatorname{ltm} K$, two of which appear to be functional orthologues of paxG and paxM from Penicillium paxilli, genes shown to encode enzymes required for early steps in paxilline biosynthesis (Fig. 6). The right-hand side of ltm cluster one in $N$. lolii is flanked by a $17.2-\mathrm{kb}$ relic of a retrotransposon sequence comprised of one retro-element (Rua) inserted within another (Tahi) (Young et al. 2005). The presence of long terminal repeats flanking each element together with the presence of 5bp direct repeats at the target sites provide strong evidence that these elements are relics of Type 1 retrotransposons. This block of retrotransposon sequence is absent from Fl1 (Fig. 6). Instead ltm $K$ is linked directly to a polyketide synthetase pseudogene that is also present in Lp19. The presence of this pseudogene and additional AT-rich sequence in both strains suggests that ltmK defines the right-hand boundary of the ltm gene cluster.

The second $l t m$ gene cluster contains five genes, $\operatorname{ltm} P, \operatorname{ltm} Q$, $\operatorname{ltm} F, \operatorname{ltm} C$ and $\operatorname{ltm} B$, four of which appear to be orthologues of the functionally characterised $\operatorname{pax} P, \operatorname{pax} Q, \operatorname{pax} C$ and $\operatorname{pax} B$ genes from P. paxilli (McMillan et al. 2003; Saikia et al. 2006; Young et al. 2001). Cluster two is separated from cluster one by a block of AT-rich retrotransposon relic sequence of approximately 35and 32-kb respectively, in Lp19 and F11 (Fig. 5).

The third ltm gene cluster contains just two genes, ltmE and ltmJ, that appear to be unique to the epichloë endophytes, and therefore lolitrem biosynthesis. A 16-kb AT-rich sequence, that includes two imperfect direct repeats, separates cluster two from cluster three. The left-hand side of cluster three is comprised of a further AT-rich sequence. Whether $\operatorname{ltm} E$ defines the left-hand boundary of the LTM locus remains to be determined, as no additional linked sequence has been cloned for analysis.

Based on the demonstration that just four gene products, PaxG, PaxM, PaxB and PaxC, are required for the biosynthesis of paspaline, the first stable indole-diterpene intermediate in paxilline biosynthesis, Young et al. (2006) proposed that the gene products of $\operatorname{ltm} G, \operatorname{ltm} M, \operatorname{ltm} B$ and $l \mathrm{tm} C$, are likely to catalyse identical steps in N. lolii and E. festucae. The fact that both ltmM and $\operatorname{ltm} C$ have been shown by complementation experiments to be functional orthologues of paxM and paxC supports this hypothesis. The inability of a symbiotum containing an $E$. festucae ltmM mutant to synthesise lolitrem B or any other indole-diterpenes confirmed that $\operatorname{tm} M$ is required for lolitrem B biosynthesis (Young et al. 2005). LtmG is proposed to catalyse the synthesis of geranylgeranyl diphosphate, the first step in lolitrem biosynthesis. GGPP then condenses with indole-3glycerol phosphate to form 3-geranylgeranylindole, a linear intermediate that has been shown by radio-labelling feeding experiments to be incorporated into paxilline (Fueki et al. 2004). LtmM is proposed to catalyse the epoxidation of the two terminal alkenes of the geranylgeranyl moiety, which is then cyclised by LtmC, to paspaline.

A comparison of the structure of lolitrem $\mathrm{B}$ with paxilline would suggest that LtmP and LtmQ, homologues of PaxP and 
PaxQ, catalyse analogous biosynthetic steps in N. lolii and E. festucae. $\mathrm{LtmP}$ is proposed to catalyse the demethylation of $\mathrm{C}-12$ of paspaline and subsequent hydroxylation of C-10, and LtmQ is proposed to hydroxylate the $\mathrm{C}-13$ position of the paspaline ring (McMillan et al. 2003; Young et al. 2006). Given the diversity of indole-diterpenes identified in $N$. lolii-infected perennial ryegrass tissue (Parker \& Scott 2004), several compounds including 13desoxypaxilline, terpendole $\mathrm{E}$, and lolicine $\mathrm{A}$, are all potential substrates for LtmQ.

Formation of the A- and B-rings of lolitrem B requires prenylation of positions 20 and 21 of the indole ring of paspaline (Fig. 7). A candidate enzyme for one or both of these prenylations is $\mathrm{LtmE}$, given the domain structure of this protein, which appears to be a fusion of two prenyl transferases (Young et al. 2006). To complete the oxidation and closure of ring-A of lolitrem B requires two additional catalytic steps. LtmJ, a P450 monooxygenase is a candidate enzyme for this step (Takemoto et al. unpublished results). At least two additional catalytic steps are required to form the epoxide between $\mathrm{C}-11$ and $\mathrm{C}-12$ of paspaline, and to oxidize and prenylate $\mathrm{C}-10$ to allow formation of ring-I of lolitrem B (Fig. 7). Candidate enzymes for the latter reactions are LtmF and LtmK (Takemoto et al. unpublished results).

The chemical diversity of indole-diterpenes identified in $N$. lolii-infected perennial ryegrass seed indicates that the A and B rings can form independently of the I ring (Gatenby et al. 1999; Munday-Finch et al. 1998). This observation led these authors to propose that lolitrem biosynthesis is modular, proceeding by way of a metabolic grid rather than a linear pathway. Chemical analysis of the metabolites that accumulate in E. festucaeperennial ryegrass symbiota containing deletions of each of the F11 ltm genes will identify the major and minor biosynthetic pathways that comprise this metabolic grid. The cloning of these genes has also allowed us to develop a PCR-based test to predict the indole-diterpene biosynthetic capability of all epichloë endophytes (Scott et al. 2007).

\section{Regulation of ltm gene expression in planta}

The $\operatorname{ltm} M$ gene was selected to examine ltm gene expression patterns in planta using deletion analysis of the $\operatorname{ltm} M$ promoter region. Five different $l t m M$ promoter deletion fragments were generated via PCR and the fragments translationally fused to gusA. The deletion constructs were then transformed into E. festucae protoplasts and the transformants tested for gusA expression using a seedling assay. The minimum promoter length required for gusA expression in planta was found to be $800 \mathrm{bp}$.

In mature vegetative tillers, gusA was expressed in all infected aerial plant tissues, including epiphyllous hyphae (Fig. 8A). The gus A expression pattern of the positive control, a Pgpd-gusA transformant, was similar to the PltmM-gusA transformants, confirming that ltmM is expressed at all times in vegetative tillers. At pre-anthesis, gusA expression was observed in all floral organs except the immature gynoecium (Fig. 8B). Similar GUS activity patterns were observed in control plants, except the ovule was colonised, with hyphae restricted to the nucellar tissue. In postanthesis florets, gene expression occurred almost exclusively in the anthers and the gynoecium with dense staining observed in the stigma (Fig. 8C). In contrast, for the control plants, GUS activity was observed in all tissues of the spikelet. In germinating seeds and seedlings, PltmM expression was observed in hyphae of seeds $24 \mathrm{~h}$ post-imbibition and from 6 days post-germination in seedlings (Fig. 8D). From 6 days, GUS activity was observed in the mesocotyl to the tip of the emerging first leaf. No GUS activity was observed in seedlings 2 to 5 days post-imbibition. These results suggest that during seed germination, only hyphae in the shoot apex have a significant role in further colonisation of the seedling.

\section{ACKNOWLEDGEMENTS}

This research was supported by grants from the Tertiary Education Commission (National Centre for BioProtection Centre of Excellence), the Royal Society of New Zealand Marsden Fund (MAU103 and MAU0403) and the New Zealand Foundation for Research, Science and Technology (C10X0203). The authors thank Brian Tapper (AgResearch) for the peramine analysis, Andrea Bryant and Ruth Wrenn (Massey University), and Michael Christensen, Wayne Simpson and Anouck De Bonth (AgResearch), for technical assistance.

\section{REFERENCES}

Ball, O.J.-P.; Miles, C.O.; Prestidge, R.A. 1997. Ergopeptine alkaloids and Neotyphodium lolii-mediated resistance in perennial ryegrass against adult Heteronychus arator (Coleoptera: Scarabaeidae). Journal of Economic Entomology 90: 1382-1391.

Ball, O.J.-P.; Prestidge, R.A.; Sprosen, J.M. 1995. Interrelationships between Acremonium lolii, peramine, and lolitrem B in perennial ryegrass. Applied and Environmental Microbiology 61: 1527-1533.

Barker, D.J.; Davies, E.; Lane, G.A.; Latch, G.C.M.; Nott, H.M.; Tapper, B.A. 1993. Effect of water deficit on alkaloid concentrations in perennial ryegrass endophyte associations. pp. 67-71. In: Proceedings of the Second International Symposium of Acremonium/Grass Interactions. Eds. Hume, D.E.; Latch, G.C.M.; Easton, H.S. AgResearch, Palmerston North, New Zealand.

Bryant, M.K.; May, K.J.; Bryan, G.T.; Scott, B. 2007. Functional analysis of a $\beta-1,6$-glucanase gene from the grass endophytic fungus Epichloë festucae. Fungal Genetics and Biology doi:10.1016/j.fgb.2006.12.012.

Bundock, P.; den Dulk-Ras, A.; Beijersbergen, A.; Hooykaas, P.J.J. 1995. Trans-kingdom T-DNA transfer from Agrobacterium tumefaciens to Saccharomyces cerevisiae. European Molecular Biology Organization Journal 14: 32063214.

Christensen, M.J.; Ball, O.J.-P.; Bennett, R.J.; Schardl, C.L. 1997. Fungal and host genotype effects on compatibility and vascular colonization by Epichloë festucae. Mycological Research 101: 493-501.

Christensen, M.J.; Bennett, R.J.; Schmid, J. 2002. Growth of Epichloë/Neotyphodium and p-endophytes in leaves of Lolium and Festuca grasses. Mycological Research 106: 93-106.

Clay, K. 1990. Fungal endophytes of grasses. Annual Review of Ecology and Systematics 21: 275-297.

de Groot, M.J.A.; Bundock, P.; Hooykaas, P.J.J.; Beijersbergen, A. 1998. Agrobacterium tumefaciens-mediated transformation of filamentous fungi. Nature Biotechnology 16: 839-842.

di Menna, M.E.; Waller, J.E. 1986. Visual assessment of seasonal changes in amount of mycelium of Acremonium loliae in leaf sheaths of perennial ryegrass. New Zealand Journal of Agricultural Research 29: 111-116.

Diebold, B.A.; Bokoch, G.M. 2001. Molecular basis for Rac2 regulation of phagocyte NADPH oxidase. Nature Immunology 2: 211-215.

Easton, H.S. 1999. Endophyte in New Zealand ryegrass pastures, 
an overview. pp. 1-9. In: Ryegrass Endophyte: An Essential New Zealand Symbiosis. Grasslands Research and Practice Series No. 7. Eds. Woodfield, D.R.; Matthew, C. New Zealand Grassland Association.

Easton, H.S.; Latch, G.C.M.; Tapper, B.A.; Ball, O.J.-P. 2002. Ryegrass host genetic control of concentrations of endophytederived alkaloids. Crop Science 42: 51-57.

Fletcher, L.R.; Easton, H.S. 1997. The evaluation and use of endophytes for pasture improvement. pp 209-227 In: Neotyphodium/Grass Interactions. Eds. Bacon, C.W.; Hill, N.S. Plenum Press, New York.

Fueki, S.; Tokiwano, T.; Toshima, H.; Oikawa, H. 2004. Biosynthesis of indole diterpenes, emindole, and paxilline: involvement of a common intermediate. Organic Letters 6: $2697-2700$

Gatenby, W.A.; Munday-Finch, S.C.; Wilkins, A.L.; Miles, C.O. 1999. Terpendole $\mathrm{M}$, a novel indole-diterpenoid isolated from Lolium perenne infected with the endophytic fungus Neotyphodium lolii. Journal of Agricultural and Food Chemistry 47: 1092-1097.

Keogh, R.G. 1973. Induction and prevention of ryegrass staggers in grazing sheep. New Zealand Journal of Experimental Agriculture 1: 55-57.

Kuldau, G.A.; Tsai, H.-F.; Schardl, C.L. 1999. Genome sizes of Epichloë species and anamorphic hybrids. Mycologia 91: 776-782.

Lambeth, J.D. 2004. NOX enzymes and the biology of reactive oxygen. Nature Reviews Immunology 4: 181-189.

Lane, G.A.; Christensen, M.J. Miles, C.O. 2000. Coevolution of fungal endophytes with grasses: the significance of secondary metabolites. pp. 341-388 In: Microbial Endophytes. Eds. Bacon, C.W.; White, J.F. Jr., Marcel Dekker, New York.

Lane, G.A.; Tapper, B.A.; Davies, E.; Hume, D.E.; Latch, G.C.M.; Barker, D.J.; Easton, H.S.; Rolston, M.P. 1997. Effect of growth conditions on alkaloid concentrations in perennial ryegrass naturally infected with endophyte. pp 179-182 In: Neotyphodium/Grass interactions. Eds. Bacon, C.W.; Hill, N.S. Plenum Press, New York.

Latch, G.C.M. 1994. Influence of Acremonium endophytes on perennial ryegrass improvement. New Zealand Journal of Agricultural Research 37: 311-318.

Latch, G.C.M.; Christensen, M.J. 1985. Artificial infection of grasses with endophytes. Annals of Applied Biology 107: 17-24.

Leuchtmann, A.; Schardl, C.L.; Siegel, M.R. 1994. Sexual compatibility and taxonomy of a new species of Epichlö symbiotic with fine fescue grasses. Mycologia 86: 802-812.

McMillan, L.K.; Carr, R.L.; Young, C.A.; Astin, J.W.; Lowe, R.G.T.; Parker, E.J.; Jameson, G.B.; Finch, S.C.; Miles, C.O.; McManus, O.B.; Schmalhofer, W.A.; Garcia, M.L.; Kaczorowski, G.J.; Goetz, M.; Tkacz, J.S.; Scott, B. 2003. Molecular analysis of two cytochrome P450 monooxygenase genes required for paxilline biosynthesis in Penicillium paxilli, and effects of paxilline intermediates on mammalian maxi-K ion channels. Molecular Genetics and Genomics 270: 9-23.

Michielse, C.B.; Hooykaas, P.J.J.; van den Hondel, C.A.M.J.J.; Ram, A.F.J. 2005. Agrobacterium-mediated transformation as a tool for functional genomics in fungi. Current Genetics 48 : $1-17$.

Munday-Finch, S.C.; Wilkins, A.L.; Miles, C.O. 1998. Isolation of lolicine A, lolicine B, lolitriol, and lolitrem N from Lolium perenne infected with Neotyphodium lolii and evidence for the natural occurrence of 31-epilolitrem $\mathrm{N}$ and 31-epilolitrem F. Journal of Agricultural and Food Chemistry 46: 590-598.
Panaccione, D.G. 1996. Multiple families of peptide synthetase genes from ergopeptine-producing fungi. Mycological Research 100: 429-436.

Panaccione, D.G.; Johnson, R.D.; Wang, J.; Young, C.A.; Damrongkool, P.; Scott, B.; Schardl, C.L. 2001. Elimination of ergovaline from a grass-Neotyphodium endophyte symbiosis by genetic modification of the endophyte. Proceedings of the National Academy of Sciences (USA) 98: 12820-12825.

Parker, E.J.; Scott, D.B. 2004. Indole-diterpene biosynthesis in ascomycetous fungi. pp. 405-426. In: Handbook of Industrial Mycology. Ed. An, Z. Marcel Dekker, New York.

Philipson, M.N.; Christey, M.C. 1986. The relationship of host and endophyte during flowering, seed formation, and germination of Lolium perenne. New Zealand Journal of Botany 24: 125-134.

Prestidge, R.A.; Gallagher, R.T. 1988. Endophyte fungus confers resistance to ryegrass: Argentine stem weevil larval studies. Ecology and Entomology 13: 429-435.

Rasmussen, S.; Parsons, A.J.; Bassett, S.; Christensen, M.J.; Hume, D.E.; Johnson, L.J.; Johnson, R.D.; Simpson, W.R.; Stacke, C.; Voisey, C.R.; Xue, H.; Newman, J.A. 2006. High nitrogen supply and carbohydrate content reduce fungal endophyte and alkaloid concentration in Lolium perenne. New Phytologist 173: 787-797

Rowan, D.D. 1993. Lolitrems, peramine and paxilline: mycotoxins of the ryegrass/endophyte interaction. pp. 103122. In: Agriculture, Ecosystems \& Environment. Elsevier Science Publishers B.V., Amsterdam.

Rowan, D.D.; Dymock, J.J.; Brimble, M.A. 1990. Effect of fungal metabolite peramine and analogs on feeding and development of Argentine stem weevil (Listronotus bonariensis). Journal of Chemical Ecology 16: 1683-1695.

Rowan, D.D.; Gaynor, D.L. 1986. Isolation of feeding deterrents against Argentine stem weevil from ryegrass infected with the endophyte Acremonium loliae. Journal of Chemical Ecology 12: 647-658.

Saikia, S.; Parker, E.J.; Koulman, A.; Scott, B. 2006. Four gene products are required for the fungal synthesis of the indole diterpene paspaline. FEBS Letters 580: 1625-1630.

Schardl, C.L.; Clay, K. 1997. Evolution of mutalistic endophytes from plant pathogens. pp. 221-238. In: The Mycota V. Plant Relationships. Part 3. Eds. Carroll, G.C.; Tudzynski, P. Springer-Verlag, Berlin Heidelberg.

Schiestl, R.H.; Petes, T.D. 1991. Integration of DNA fragments by illegitimate recombination in Saccharomyces cerevisiae. Proceedings of the National Academy of Sciences (USA) 88: 7585-7589.

Scott, B.; Young, C.A.; Tapper, B.A.; Wrenn, R.E.; Foster, S.J.; Moon, C.D.; Schardl, C.L. 2007. Peramine and indolediterpene biosynthetic capability of epichloë endophytes as predicted by perA and $\mathrm{ltm}$ gene analysis. In: Proceedings of the $6^{\text {th }}$ International Symposium on Fungal Endophytes of Grasses. Grassland Research and Practice Series No. 13. Eds. Popay, A.J; Thom, E.R. New Zealand Grassland Association Publication.

Siegel, M.R.; Latch, G.C.M.; Bush, L.P.; Fannin, F.F.; Rowan, D.D.; Tapper, B.A.; Bacon, C.W.; Johnson, M.C. 1990. Fungal endophyte-infected grasses: alkaloid accumulation and aphid response. Journal of Chemical Ecology 16: 3301-3315.

Spiering, M.J.; Lane, G.A.; Christensen, M.J.; Schmid, J. 2005. Distribution of the fungal endophyte Neotyphodium lolii is not a major determinant of the distribution of fungal alkaloids in Lolium perenne plants. Phytochemistry 66: 195-202. 
Takemoto, D.; Tanaka, A. Scott, B. 2006. A p67 Phox-like regulator is recruited to control hyphal branching in a fungal-grass mutualistic symbiosis. The Plant Cell 18: 2807-2821.

Tan, Y.Y.; Spiering, M.J.; Scott, V.; Lane, G.A.; Christensen, M.J.; Schmid, J. 2001. In planta regulation of extension of an endophytic fungus and maintenance of high metabolic rates in its mycelium in the absence of apical extension. Applied and Environmental Microbiology 67: 5377-5383.

Tanaka, A.; Christensen, M.J.; Takemoto, D.; Park, P.; Scott, B. 2006. Reactive oxygen species play a role in regulating a fungus-perennial ryegrass mutualistic association. The Plant Cell 18: 1052-1066.

Tanaka, A.; Tapper, B.A.; Popay, A.; Parker, E.J.; Scott, B. 2005. A symbiosis expressed non-ribosomal peptide synthetase from a mutualistic fungal endophyte of perennial ryegrass confers protection to the symbiotum from insect herbivory. Molecular Microbiology 57: 1036-1050.

Tanaka, A.; Wrenn, R.E.; Takemoto, D.; Scott, B. 2007. Agrobacterium tumefaciens T-DNA mediated transformation of Epichloë festucae. In: Proceedings of the $6^{\text {th }}$ International Symposium on Fungal Endophytes of Grasses. Grassland Research and Practice Series No. 13. Eds. Popay, A.J; Thom,
E.R. New Zealand Grassland Association Publication.

Walton, F.J.; Idnurm, A.; Heitman, J. 2005. Novel gene functions required for melanization of the human pathogen Cryptococcus neoformans. Molecular Microbiology 57: 1381-1396.

Wang, J.; Machado, C.; Panaccione, D.G.; Tsai, H.-F.; Schardl, C.L. 2004. The determinant step in ergot alkaloid biosynthesis by an endophyte of perennial ryegrass. Fungal Genetics and Biology 41: 189-198.

Young, C.; McMillan, L.; Telfer, E.; Scott, B. 2001. Molecular cloning and genetic analysis of an indole-diterpene gene cluster from Penicillium paxilli. Molecular Microbiology 39: 754-764.

Young, C.A.; Bryant, M.K.; Christensen, M.J.; Tapper, B.A.; Bryan, G.T.; Scott, B. 2005. Molecular cloning and genetic analysis of a symbiosis-expressed gene cluster for lolitrem biosynthesis from a mutualistic endophyte of perennial ryegrass. Molecular Genetics and Genomics 274: 13-29.

Young, C.A.; Felitti, S.; Shields, K.; Spangenberg, G.; Johnson, R.D.; Bryan, G.T.; Saikia, S.; Scott, B. 2006. A complex gene cluster for indole-diterpene biosynthesis in the grass endophyte Neotyphodium lolii. Fungal Genetics and Biology 43: 679-693. 